Berichtigung

Klin. Wochenschr. 57, 1097-1107 (1979)

Tönnesmann, E., Bürkle, P.A., Schäfer, B., Federlin, K.

\title{
Humoraler und zellulärer Immunstatus bei Patienten mit Morbus Crohn
}

In obiger Arbeit sind zwei Druckfehler zu berichtigen:

1. Seite 1100 (Tabelle 2) n 4

$+++++$

2. Seite 1104 (rechte Spalte, letzte Zeile) ... bisher nur

anbehandelte Patienten ...

\section{Buchbesprechungen}

Barker Jorgensen, C., Skadhauge, E.: Alfred Benzon Symposium XI. Osmotic and Volume Regulation. Copenhagen: Munksgaard 1977. 512 pages.

Der vorliegende Bericht eines Symposiums enthält eine Fülle von Informationen aus verschiedenen Disziplinen der Biologie: zur vergleichenden Physiologie und Systemanalyse der Osmo- und Volumenregulation, uber die Regulation der Wasserzufuhr (Durst), ferner zum Wirkmechanismus des antidiuretischen Hormons, die Beeinflussung der Volumenregulation durch Corticosteroide, über die Rolle verschiedener Effektororgane (Niere, Darmtrakt) sowie Messungen der intra-extrazellulären Wasserverteilung in Kalt- und Warmblüterzellen. Ungekürzte Wiedergabe der Vortragstexte, zahlreiche Schrifttumshinweise und sorgfältig redigierte Diskussion zu jedem einzelnen Vortrag, umfangreiches Sachregister. - Das Buch ist eine einzigartige und auch wegen seiner Qualität wertvolle Quelle neuer experimenteller Forschungsergebnisse und gehört in die Bibliotheken der biologischen und physiologischen Institute bzw. nephrologischen Abteilungen.

G. Riecker (München)

Fein, J.M., Reichmann. O.H.: Microvascular Anastomoses for Cerebral Ischemia. Berlin, Heidelberg, New York: Springer 1978. XV, $324 \mathrm{~S}$. mit $197 \mathrm{Abb}$. incl, 12 color plates. Cloth DM 96,-; US $\$ 48.00$

Das Buch setzt sich aus den auf dem 2. internationalen Symposium über mikroneurochirurgische Anastomosen in der Behandlung der cerebrovaskulären Insuffizienz im Jahre 1974 in Chicago gehaltenen Vorträge zusammen. Die klinischen und experimentellen Arbeiten sind in 7 Kapitel unterteilt. Obwohl der Gehalt nützlicher Informationen in den einzelnen Arbeiten unterschiedlich ist, was für ein Muttiautoren-Buch unvermeidlich ist, bleibt die wissenschaftliche Qualität überdurchschnittlich hoch. Gleichzeitig zeichnet sich das Buch durch eine gute Wiedergabe des bildlichen Materials aus. Das 1. Kapitel - Struktur und Funktion - beinhaltet experimentelle Arbeiten, die sich mit der Angioarchitektur des Carotis- und vertebro-basilären Systems, mit Modellen zum Studium der cerebrovaskulären Ischämie am Hund sowie mit elektronenmikroskopischen Studien über Endothelveränderungen nach mikrochirurgischen Anastomosen-Operationen beschäftigen. Im 2. Kapitel - Metabolismus - werden die Effekte der cerebralen Anoxie und Ischämie auf den oxydativen Metabolismus und ihre Veränderungen nach der extraintrakraniellen Bypass-Operation sowie die Mechanismen des protektiven Effektes der Barbiturate für die cere- brovaskuläre Insuffizienz erörtert. In den folgenden 5 Kapiteln klinische Beurteilung und Diagnose, chirurgische Technik, klinischhämodynamische Aspekte, klinische Ergebnisse und andere Applikationen - werden klinische, diagnostische und mikroneurochirurgisch-therapeutische Aspekte der cerebrovaskulären Ischämie abgehandelt. Als besonders wertvoll heben sich hier die Beiträge über prä- und postoperative Messungen der quantitativen regionalen Hirndurchblutung sowie über zwei verschiedene Methoden zur Flow-Messung nach Anastomosenoperationen ab. Obwohl das Buch erst 4 Jahre nach dem Symposium erscheint, ist es hauptsächlich für Neurologen, Neurochirurgen und Internisten, die sich für die Behandlung der cerebrovaskulären Ischämie interessieren, von besonderem Anschaffungswert. V. Olteanu-Nerbe (München)

Gremmel, H., Kaiser, K., Löhr, H.H., Vieten, H.: Röntgendiagnostik des Herzens und der Gefäbe. (Handbuch der Medizinischen Radiologie. Bd. 10, T.2c.) Berlin, Heidelberg. New York: Springer 1979. $\mathrm{X}, 421 \mathrm{~S}$. mit $102 \mathrm{Abb}$. in 252 Einzeldarst. Geb. DM 480,-; US- $\$ 264.00$

Der vorliegende zehnte Handbuchband behandelt die offenen und stumpftraumatischen Verletzungen, einschließlich Fremdkörperverletzungen des Herzens und der großen herznahen Gefäße. Die Diagnosestellung derartiger Verletzungen ist in der Hand des Radiologen zu einer wichtigen und verantwortungsvollen Aufgabe geworden, ergibt sich doch gerade in der heutigen Verkehrssituation durch die zahlreichen Autounfälle mit ihren Schub- und Schleuderwirkungen zunehmend ein Unfallmechanismus, der zu offenen oder stumpfen Thoraxtraumen führt. - Ebenso kommt es iatrogen durch die in steigendem $\mathrm{MaBe}$ angewendeten invasiven cardiologisch-angiographischen Untersuchungstechniken wie auch infolge von Operationen am offenen Herzen zu Verletzungsfolgen am Herzen und den großen Gefäßen: Perforationen, Fremdkörper (abgebrochene Katheterteile), Herztamponade, postoperative Aneurysmen etc. - Hier kann nur rasches Erkennen des Krankheitsbildes unter Einsatz aller zur Verfügung stehender röntgendiagnostischer Möglichkeiten dem Patienten helfen. Dabei spielen konventionelle röntgendiagnostische Verfahren mit für die Unfallchirurgie geeigneten technisch-apparativen Ausrüstungen eine wichtige Rolle, aber auch Angiocardiographie, nuklearmedizinische Techniken und zunehmend auch die Computertomographie. - Ein umfassendes Bildmaterial dokumentiert die traumatisch bedingten Herz- und Gefäßveränderungen im Röntgenbild. In sehr anschau- 